\title{
Age-related differences in muscular capacity among workers
}

\author{
Heleen H. Hamberg-van Reenen • Allard J. van der Beek • \\ Birgitte M. Blatter · Willem van Mechelen • \\ Paulien M. Bongers
}

Received: 9 July 2008 / Accepted: 6 February 2009 / Published online: 1 March 2009

(c) The Author(s) 2009. This article is published with open access at Springerlink.com

\begin{abstract}
Purpose To quantify the age-related changes in muscular capacity in a working population, and to investigate whether these changes are dependent on sports participation.

Methods Data were used from the longitudinal study on musculoskeletal disorders, absenteeism, stress and health $(n=1,800)$. At baseline, isokinetic lifting strength and static muscle endurance were assessed, and endurance measurements were repeated after 3 years of follow-up. Sports participation was assessed using a questionnaire.

Results Cross-sectionally, static endurance of the neck/ shoulder muscles was highest among older workers, but decreased longitudinally among all age groups. Younger workers who participated in sports $3 \mathrm{~h}$ per week or more had the best performance, but older workers who participated between 0 and $3 \mathrm{~h}$ per week had better performance than those who participated in sports more frequently.

Conclusions There were age-related differences on muscular capacity. Younger workers who participated in sports frequently had the best muscular capacity. For aging workers,
\end{abstract}

\footnotetext{
H. H. Hamberg-van Reenen · A. J. van der Beek ( $)$.

B. M. Blatter · W. van Mechelen · P. M. Bongers

Body@Work, Research Center Physical Activity,

Work and Health, TNO-VU University Medical Center,

Van der Boechorststraat 7, 1081 BT Amsterdam, The Netherlands

e-mail: a.vanderbeek@vumc.nl
}

H. H. Hamberg-van Reenen - A. J. van der Beek .

W. van Mechelen · P. M. Bongers

Department of Public and Occupational Health,

EMGO Institute, VU University Medical Center,

Amsterdam, The Netherlands

H. H. Hamberg-van Reenen · B. M. Blatter · P. M. Bongers

TNO Quality of Life, Hoofddorp, The Netherlands moderate sports participation seems to be effective in keeping them suitable for the relatively growing work demands.

Keywords Muscle strength · Muscle endurance · Muscular capacity · Age-related differences · Aging · Workers

\section{Introduction}

Nowadays, the percentage of older workers is rising, due to increasing life expectancy, increasing retirement age, and increasing societal demand on continued participation of older workers.

The aging worker is in many aspects different from the younger worker, due to physical and mental changes associated with aging. Between the ages of 25 and 70 , the body composition changes, characterized by a doubling of the total body fat proportion, loss of muscle fibers, and bone loss (World Health Organization 1993; Macaluso and De Vito 2004). These changes lead to a decrease in muscle strength (De Zwart et al. 1995; Izquierdo et al. 2001; Macaluso and De Vito 2004; Savinainen et al. 2004b). In general, muscle strength reaches its optimum between the second and the third decade, for women a few years earlier than for men. The maximal muscle strength of a 65-year old person is on average about $75-80 \%$ of that person's lifetime maximal muscle strength (Asmussen and HeebollNielsen 1962; De Zwart et al. 1995; Ilmarinen 2001; Macaluso and De Vito 2004). Savinainen et al. (2004a) reported a decline in muscle strength of the back and arm muscles during 16 years of follow-up among middle-aged subjects.

Muscle endurance has received much less attention in the literature. Unless different physiological changes in the muscle tissue, and muscle blood flow among older subjects 
(Bemben 1998), muscle endurance was found to be unaffected by age, or even to increase with age in some studies (Alaranta et al. 1994; De Zwart et al. 1995; Bemben et al. 1996; Bemben 1998; Hunter et al. 2005). Older subjects were often found to be more muscle fatigue resistant than younger subjects when sustaining static contractions (Hunter et al. 2005).

Next to musculoskeletal changes, cardiovascular and respiratory capacity decrease with age, even at a higher degree than the decrease in muscular capacity (De Zwart et al. 1995; Era et al. 2001; Izquierdo et al. 2001; Savinainen et al. 2004b).

Inter-individual differences in the age-related changes of physical capacity are enormous among workers, due to differences in the physical activity level. Age-related declines in physical capacity can be slowed down by regular physical training (Rantanen et al. 1993; De Zwart et al. 1995; Ilmarinen 2001; Brach et al. 2004; Macaluso and De Vito 2004). However, high physical workload was not found to have a long-lasting training effect on the muscle strength of aging workers (Savinainen et al. 2004b; Ilmarinen 2001).

In several jobs, the work demands for aging workers are at the same level as for younger workers (Lusa et al. 1994; De Zwart et al. 1995; Sluiter 2006). Owing to the decreasing working capacity, the resulting workload might change from an acceptable load into daily physical "overload", which might result in long-term health effects with chronic musculoskeletal symptoms as the main effect (De Zwart et al. 1997; Seitsamo and Klockars 1997).

Most studies on age-related differences in muscle strength or static muscle endurance consisted of a small study population with a small age-range. Furthermore, few studies focused on a working population, while the agerelated decline in physical capacity has important consequences for the aging worker, because of the risk of an overload at work. In this study, we describe the age-related differences in isokinetic lifting strength and static muscle endurance of the low back, neck, and shoulder muscles in approximately 1,500 male and female workers with different professions in the Netherlands. With regard to static muscle endurance, we studied the relation with age both cross-sectionally and longitudinally with a follow-up of 3 years within the same dataset. For isokinetic lifting strength, we stratified for gender. In order to account for a potential physical training effect (Rantanen et al. 1993; De Zwart et al. 1995; Ilmarinen 2001; Brach et al. 2004; Macaluso and De Vito 2004), we also stratified for (self-reported) sports participation.

The objective of the present study is twofold: (1) to quantify the age-related (and gender-specific) differences in lifting strength and static muscle endurance in a working population, and (2) to investigate whether these are different for workers who participate in sports and those who do not.

\section{Methods}

The longitudinal study on musculoskeletal disorders, absenteeism, stress and health (SMASH) is a prospective cohort study among almost 1,800 workers from 34 different companies with a follow-up of 3 years. At baseline in 1993, we assessed muscular capacity, including isokinetic lifting strength and static muscle endurance in the low back, neck and shoulder region. After 3 years of follow-up, measurements of static muscle endurance in the low back, neck and shoulder region were repeated, but for practical reasons, lifting strength was only measured once at baseline.

We selected a study population of workers who worked at least 1 year in their current job for more than $20 \mathrm{~h}$ per week, not receiving a sickness benefit or a permanent disability pension (approximately 1,500 workers).

Measurement of isokinetic lifting strength and static muscle endurance

Trained physiotherapists performed the different tests of muscular capacity. At baseline, isokinetic lifting strength of the back and neck/shoulder muscles was measured. Both at baseline and after 3 years of follow-up, sub-maximal endurance time of static contraction of the back, neck and shoulder muscles was measured.

Isokinetic lifting strength of the low back and neck/ shoulder muscles was measured using the Aristokin dynamometer (Lode BV Medical Technology, Groningen, the Netherlands). The lifting strength was measured during three lifting movements with maximum effort and a velocity of $40 \mathrm{~cm} / \mathrm{s}$ with a rest period of $30 \mathrm{~s}$ in between, both standardized movements upright from floor to hip level, and from hip to shoulder level. Isokinetic lifting strength (in Newtons) was defined as the average outcome of the second and third lift.

Static endurance of the back, neck and shoulder muscles was defined as the number of seconds during which the workers could keep a position, while carrying a gender-specific load (maximized at 240 and $420 \mathrm{~s}$, for the low back and the neck/shoulder regions, respectively). The BieringSørensen test (1984) was used for the back extensors. During this test, workers were lying prone on a table and had to keep their unsupported upper part of the body in a horizontal position with fixation of the buttocks and legs. For the measurement of the static endurance of the neck extensors, the workers had to keep their head flexed in a sitting position, while carrying a loaded helmet of $5 \mathrm{~kg}$ for males and $2.5 \mathrm{~kg}$ for females. For the measurement of the static endurance of the shoulder elevators, workers had to keep their arms elevated at $90^{\circ}$ in a sitting position, while carrying a load of $2.5 \mathrm{~kg}$ for males and $1.5 \mathrm{~kg}$ for females. The endurance tests were finished when a discomfort rating of 5 in the 
test region or a score of 7 in another part of the body (on a 10-point Borg scale) was reported (Borg 1990; Van der Grinten 1992).

Workers with contraindications (such as cardiovascular diseases, fever or pregnancy) that might involve a health risk, or that might have an effect on the results of the tests, were excluded from the physical capacity tests. In addition, workers who reported a discomfort rating of 4 or higher before the start of the test were excluded from the tests. Further details on the different tests of muscular capacity were described elsewhere (Hamberg-van Reenen et al. 2006).

\section{Assessment of sports participation}

Data on sports participation were assessed using a questionnaire at baseline. The workers were asked for physically demanding sports during the preceding 12 months. Those who never participated in sports in that year were distinguished from those who did participate in sports. Furthermore, a distinction in frequency was made, i.e. participation for $3 \mathrm{~h}$ per week or more and participation less than $3 \mathrm{~h}$ per week.

\section{Data analyses}

We analyzed the course of static muscle endurance by age both cross-sectionally and longitudinally during the followup period of 3 years. To take account of potentially mathematically parabolic relations with age, we analyzed the cross-sectional data using quadratic regression analyses. We added a squared age term as an independent variable to the regression functions. To correct for the dependency of age and squared age, we used the square of age minus mean age (Cohen 2003). Longitudinally, we analyzed the mean differences in static muscle endurance time at baseline and after 3 years of follow-up for 5-year age groups. This was presented as lines from the middle of the 5-year age groups at baseline to the middle of the 5-year age groups 3 years later. The number of workers for the longitudinal analyses was smaller than the number of workers for the cross-sectional analyses, due to loss to follow-up. Furthermore, cross-sectionally, we presented stratified results for frequency of sports participation (i.e. never, $<0$ and $<3$, and $\geq 3 \mathrm{~h}$ ). Finally, for isokinetic lifting strength, we analyzed stratified regression functions for sports participation and gender. To analyze to what extent muscular capacity was statistically significantly different for gender- and sportgroups, we added interaction terms to the regression functions. We presented $R^{2}$ and regression functions $(a, b 1$ and $b 2$ ) in addition to the graphics of the regression functions.

\section{Results}

Almost $70 \%$ of the workers were male. At baseline, the mean age was 35 years ( 37 years among men, and 33 years among women); the youngest worker had an age of 19 and the oldest an age of 59. Figure 1 shows the age distribution of the study population $(n=1,578)$.

Figure 2 presents the course of static muscle endurance time according to age. This figure presents both the crosssectional relations at baseline (continuous lines), and the mean differences between baseline and follow-up for different age groups (longitudinal analyses represented by the lines between upper dots at baseline and lower dots after 3 years of follow-up at the middle of the age groups). Cross-sectionally, the mean performance for static endurance time of the back muscles had its optimum at the age of 36 years, with $85 \%$ of that optimum at the age of 59 years. For the neck and shoulder muscles, static muscle endurance time at the age of 59 years was 2.0 and 1.5 times higher, respectively, than static muscle endurance time at the age of 19 years. In contrast, from the longitudinal analyses, it can be seen that static muscle endurance time of the back, neck and shoulder muscles decreased statistically significantly $(P \leq 0.05)$ among all age groups with values of $77 \%$ on average after three years of follow-up compared with the
Fig. 1 Age distribution of the SMASH working population $(n=1,578)$

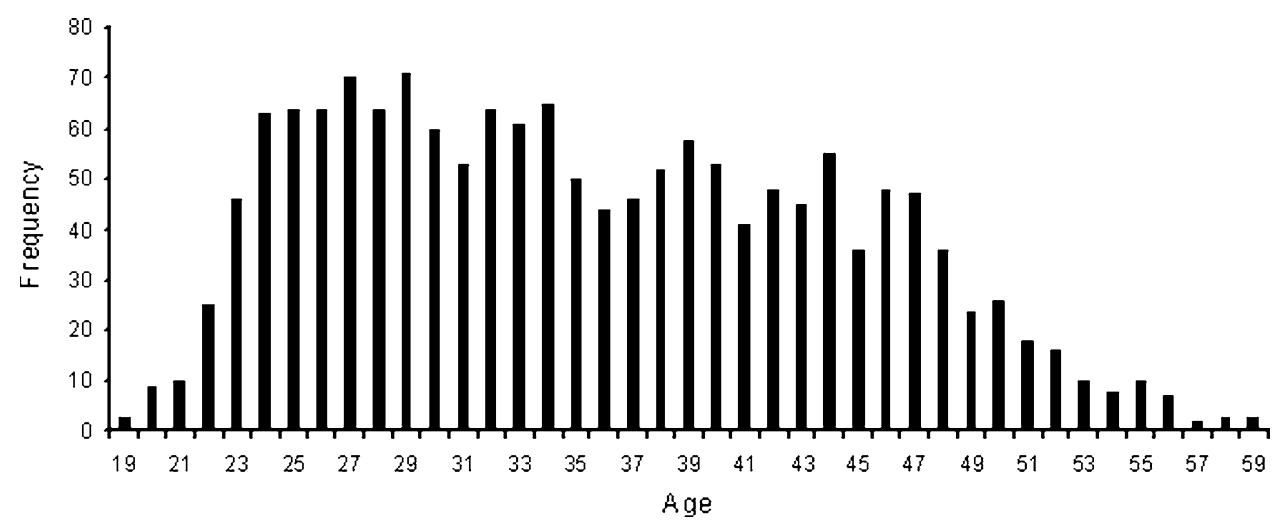


Fig. 2 Cross-sectional regression functions of baseline static muscle endurance time of the back muscles a the neck muscles and $\mathbf{b}$ the shoulder muscles $\mathbf{c}$ by age. Longitudinal means by age groups at baseline [upper dots at the middle of the age groups (19-24 to 54-59 years)] and after 3 years of follow-up [lower dots at the middle of the age groups (22-27 to 57-62 years)]

Fig. 3 Cross-sectional regression functions of baseline static muscle endurance time of the back muscles (a), the neck muscles (b) and the shoulder muscles (c) by age. Stratified for sports participation: never (continuous lines), $>0$ and $<3 \mathrm{~h}$ per week (large dotted lined), and $\geq 3 \mathrm{~h}$ per week (small dotted lines)
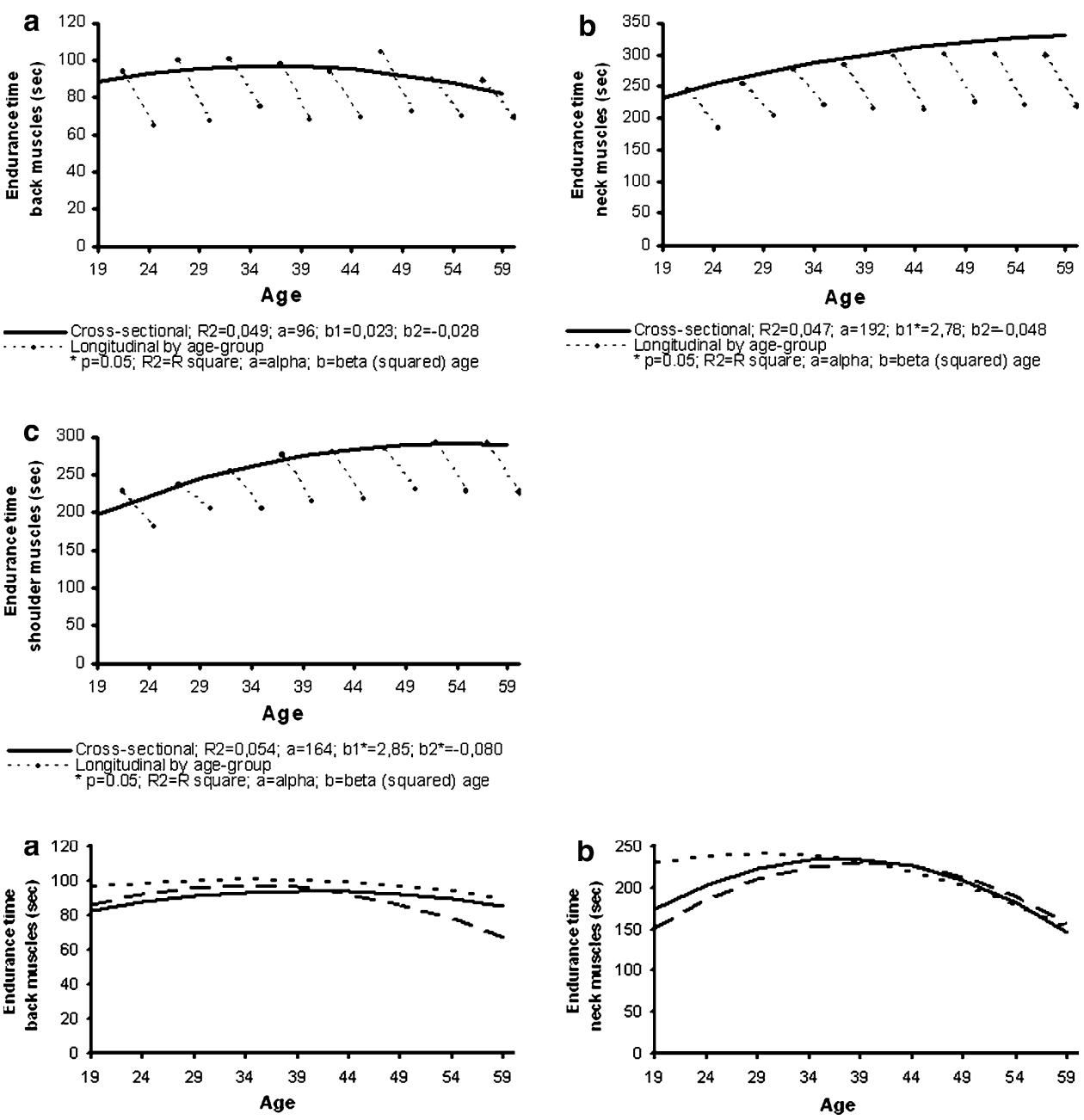

- Never sporting; $R 2=0,003 ; a=85 ; b 1=0,23 ; b 2=-0,025$ - - - Sporting $<=3 \mathrm{hrSivk;}$ R2 $2=0,008 ; a=102 ; b 1=-0,14 ; b 2=-0,049$

- Never sporting; $R 2=0,036 ; a=198 ; b 1 *=2,54 ; b 2=-0,059$ - - Sporting $<=3$ hrSivk; R2=0,070; $a=162 ; b 1^{*}=3,48 b 2=0,024$

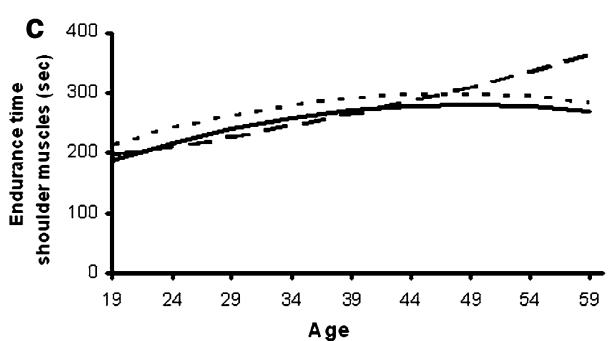

- Never sporting; $R 2=0,046 ; a=166 ; b 1^{*}=2,72 ; b 2^{*}=-0,11$

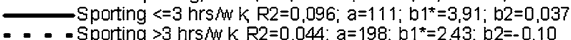

baseline values. The $R^{2}$ is 0.05 or lower, which means that $5 \%$ or less of the variation in static endurance time can be explained by age.

Figure 3 shows baseline static muscle endurance time by age stratified for sports participation. It can be seen that there were only small differences between the sports participation groups. Younger workers who participated in sports for at least $3 \mathrm{~h}$ per week had the longest endurance time. There are only small differences between workers who participate in sports for fewer hours per week or not at all. For older workers, either frequently sporting workers (for the back muscles) or moderate frequently sporting workers (for the shoulder muscles) had the longest endurance time or the endurance time is equal for sporting or not sporting workers (for the neck muscles). Ten percent or less of the variation in static endurance time can be explained by age $\left(R^{2}\right.$ between 0.001 and 0.10 ).

Figure 4 presents baseline isokinetic lifting strength by age among men and women stratified for three groups with regard to sports participation. Isokinetic lifting strength of 
Fig. 4 Cross-sectional regression functions of isokinetic lifting strength by age $\mathbf{a}$ of the back muscles and $\mathbf{b}$ the neck/shoulder muscles. Stratified for sports participation: never (continuous lines), $>0$ and $<3 \mathrm{~h}$ per week (large dotted lined), and $\geq 3 \mathrm{~h}$ per week (small dotted lines)

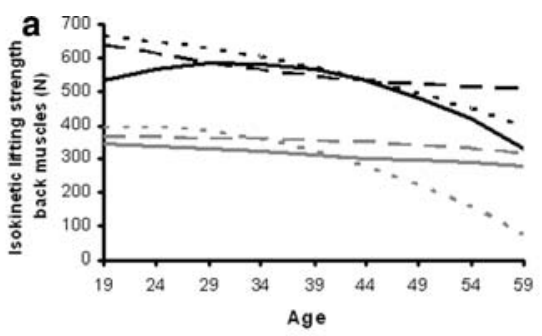

C - - Men; sparting $<=3 \mathrm{hrS} / \mathrm{W} \mathrm{K} \quad \mathrm{R} 2=0,017 ; \mathrm{a}=693 ; \mathrm{b} 1=3,75 ; \mathrm{b} 2=0,06$ - - Men; sporting $>3$ hrs $/$ W K R2 $=0,049, a=806 ; b 1 *=6,00 ; b 2=0,10$ Women never sporting $\mathrm{R}_{2}=0,018 ; \mathrm{a}=376 ; \mathrm{b} 1=-1,63 ; \mathrm{b} 2=-0,01$ - Wornen; sporting $c=3 \mathrm{hrs} / \mathrm{W}$ K R2 $=0,003 ; a=389 ; \mathrm{b} 1=0,89 ; \mathrm{b} 2=-0,03$ - - Women; sporting $>3 \mathrm{hrs} / \mathrm{W}$ K $R 2=0,12, \mathrm{a}=575 ; \mathrm{b} 11^{*}=-6,35, \mathrm{~b} 2=0,22$ * $p=0$ 05: $R 2=R$ square; $a=a$ pha $b=b$ eta (squared) aqe

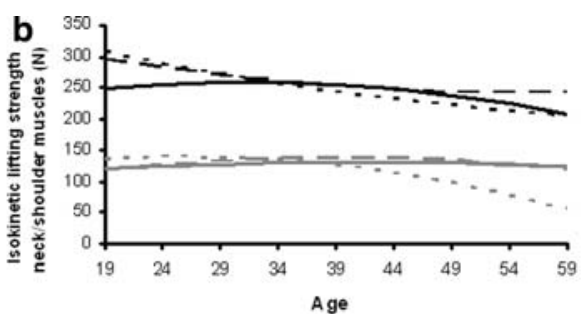

Men; never sporting; $\mathrm{R} 2=0,010 ; \mathrm{a}=277 ; \mathrm{b} 1=-0,53 ; \mathrm{b} 2=0,071$ - - Men; sporting $<=3 \mathrm{hrS} / \mathrm{W}$ K R2 $=0,020 ; \mathrm{a}=318 ; \mathrm{b} 1^{*}=-1,68 ; \mathrm{b} 2=0,043$ - - Men; sporting > $3 \mathrm{hrs} / \mathrm{W}$ k $R 2=0,061 ; a=352 ; b 1 *=2,79 ; b 2=0,033$ Women; never sporting. $R 2=0,003 ; a=121 ; b 1=0,24 ; b 2=0,020$ - - Women sporting $\Leftrightarrow=3$ hrs Aw K R2 $=0,013 ; 3=125 ; b 1=0,37, b 2=0,000$

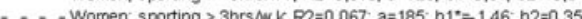
- $p=0.05 ; R 2=R$ square; $a=a l p h a, b=b e t a$ (squared) age the back and neck/shoulder muscles among the men was, respectively, 1.6 and 2.0 times higher than the isokinetic lifting strength among the women. The figure shows the highest isokinetic lifting strength among young workers who participated in sports $3 \mathrm{~h}$ per week or more, and among older workers who participated in sports less than $3 \mathrm{~h}$ per week. The differences between men and women were statistically significant ( $P$ interaction terms $<0.05$ ), but the differences between the three groups on sports participation were not statistically significant $(P$ interaction terms $>0.10)$. Of the variation in isokinetic lifting strength, $12 \%$ or less can be explained by age.

\section{Discussion}

Main results in comparison with previous research

Previous studies reported mixed results with regard to the age-related changes in muscle endurance (Alaranta et al. 1994; De Zwart et al. 1995; Bemben 1998; Hunter et al. 2005). Cross-sectionally, we found optima of static endurance time of the back muscles at the age of 36 years, However, for the neck and shoulder muscles, static muscle endurance time at the age of 59 years was between 2.0 and 1.5 times higher than at the age of 19 years. In contrast, longitudinally, we found that muscle endurance decreased for all age groups. The direction of the aging effect was opposite when comparing the cross-sectional with the longitudinal results.

With regard to performance by sports participation, the results of this study suggest that younger workers who participated in sports for 3 hours per week or more had the highest isokinetic lifting strength and the longest static muscle endurance time. This is in-line with results from previous studies (Rantanen et al. 1993; De Zwart et al. 1995; Ilmarinen 2001; Brach et al. 2004; Macaluso and De Vito 2004). As expected, we found that isokinetic lifting strength was lower at older ages than at younger ages due to the aging process. The differences by age were the largest in the group participating in sports for $3 \mathrm{~h}$ per week or more, i.e. the plotted lines crossed over between the ages of 30 and 40. Furthermore, the results suggest that older workers who participated in sports between 0 and $3 \mathrm{~h}$ per week had better performance in tests of physical capacity than those who were inactive or participated in sports for $3 \mathrm{~h}$ per week or more, which was not in-line with our expectation that the age-related differences would be smallest among the most active workers.

Possible explanations for the differences

between the cross-sectional and longitudinal results

The differences between the cross-sectional and longitudinal analyses were contrary to our expectations. Owing to a potential healthy worker effect, we expected to find equal or fewer age-related differences in within-worker comparisons compared with between-worker comparisons. However, the results suggest that there was no healthy worker effect. Several factors can explain this finding.

First, there could have been a period or measurement time effect (Twisk 2003) due to different test circumstances at follow-up compared with baseline. Possible differences in test circumstances may have been the result of less motivation of the workers during the tests, to other physiotherapists who conducted the tests or to seasonal effects. In pilot studies, reproducibility was found to be high for the isokinetic neck/shoulder lifting test and the trunk muscle endurance test and moderate for the other tests of muscular capacity (Hamberg-van Reenen et al. 2006). However, in the present study, reproducibility between the tests at baseline and follow-up was low (Spearman correlation coefficients were 0.47 for the back, 0.40 for the neck and 0.51 for the shoulders). This could be an indication of a period effect. With respect to the motivation of the workers during the tests, most workers were well motivated (on a threepoint scale) both at baseline and at follow-up. However, some were less motivated at follow-up than at baseline. Both at baseline, and at follow-up, the performance among workers who were well motivated was statistically significantly 
higher than among workers who were moderately or poorly motivated. However, the difference between performance at follow-up and at baseline was about the same for well motivated compared with poorly motivated workers. This means that changes in motivation could not explain the differences between the cross-sectional and longitudinal analyses. With respect to potential differences between the 16 physiotherapists who conducted the tests of muscular capacity, the mean performance differed statistically significantly both at baseline and at follow-up between the different physiotherapists. This was in spite of a training before the data collection, and moderate inter-rater reliability in the pilot studies (Hamberg-van Reenen et al. 2006). However, most workers were supported by a different physiotherapist at follow-up than at baseline. When comparing the difference in mean performance between follow-up and baseline with the different physiotherapist's combinations at baseline and follow-up, no association was found. Therefore, potential misclassification cannot have been differential, which means that a change in physiotherapist cannot explain the differences between the cross-sectional and longitudinal analyses. Furthermore, to find out if sports participation or physical workload during follow-up could have played a role, we did additional longitudinal analyses stratified for baseline sports participation and for baseline physical workload (defined as blue collar or white collar work). However, we found no other pattern as the non-stratified analyses: the decrease in static muscle endurance during follow-up was comparable for all groups regardless of sports participation or workload. We expected that the baseline results are a good proxy for the follow-up results, because in additional analyses on sports participation during follow-up, sports participation did not change considerable during follow-up on average. Therefore, it does not seem plausible to explain the systemic decrease in static endurance time during follow-up by a systematic decrease in sports participation or physical workload. Finally, no differences were found regarding the season of testing. For all workers, the physical tests at follow-up were assessed more or less in the same month 3 years later, with a month difference at maximum. In conclusion, because we could not confirm differences in test circumstances, other unknown factors outside the test circumstances should be sought to explain the period effect.

Second, there could have been a cohort effect (Twisk 2003), because the population in the longitudinal analyses was different from the population at baseline in the crosssectional analyses due to loss to follow-up. The loss to follow-up rates were $15 \%$ for the low back tests, $31 \%$ for the neck tests and $18 \%$ for the shoulder tests, respectively. The main reasons for loss to follow-up were general reasons, such as discharge, lack of motivation, et cetera. We investigated if this loss to follow-up could have been selective by comparing the total mean performance at baseline among workers who became lost to follow-up to those who did not become lost to follow-up. The static endurance time of the shoulder muscles at baseline was significantly shorter among those who became lost to follow-up, although the mean difference was only $3 \mathrm{~s}$ (256 compared to $259 \mathrm{~s}$ ). In contrast, we found a significantly longer static endurance time of the neck muscles for that group (305 compared to 274 s). This means that there was selective loss to followup, but the difference for the shoulder muscles was very small, and the difference for the neck muscles was not in the expected direction. Therefore, it seems unlikely that a cohort effect on muscular capacity could have played a role in the differences between the cross-sectional and the longitudinal results.

Third, the statistical analyzing techniques were different, i.e. cross-sectionally, regression analyses were used, and longitudinal, a description of repeated means was presented for 5-year age groups. However, if we had described means in the cross-sectional analyses as well, the results would have been quite the same compared with the estimated regression functions (data not shown). This means that it is unlikely that differences in statistical analyzing techniques have contributed to the differences between the cross-sectional and longitudinal results.

Finally, a comment should be made on the longitudinal results, since we had only data at two measurements with a three-year interval. Owing to this short interval, in particular compared to the duration of a general working lifetime, conclusions on the longitudinal results have to be taken with caution.

In conclusion, other factors than differences in test circumstances, selectiveness of loss to follow-up, or differences in statistical analyzing techniques have to be sought to explain the difference between cross-sectional and longitudinal results regarding the static muscles endurance.

\section{Conclusions}

The results of this study suggest age-related differences of isokinetic lifting strength, and static muscle endurance of the back and neck/shoulder muscles. For isokinetic lifting strength and static endurance of the back muscles, the performance was higher among younger workers than among older workers, but for static endurance of the neck and shoulder muscles, the age-related differences were opposite. In contrast, after three years of follow-up, decreased static muscle endurance was found for all ages. Factors other than differences in test circumstances, or loss to follow-up have to be sought to explain the differences between cross-sectional and longitudinal results with respect to static muscle endurance. 
Younger workers who participated in sports for $3 \mathrm{~h}$ per week or more had the best muscular capacity, but older workers who participated in sports between 0 and $3 \mathrm{~h}$ per week had better muscular capacity than those who were inactive or participated in sports for $3 \mathrm{~h}$ per week or more.

Open Access This article is distributed under the terms of the Creative Commons Attribution Noncommercial License which permits any noncommercial use, distribution, and reproduction in any medium, provided the original author(s) and source are credited.

\section{References}

Alaranta H, Hurri H, Heliovaara M, Soukka A, Harju R (1994) Nondynamometric trunk performance tests: reliability and normative data. Scand J Rehabil Med 26:211-215

Asmussen E, Heeboll-Nielsen K (1962) Isometric muscle strength in relation to age in men and women. Ergonomics 5:167-169. doi:10.1080/00140136208930570

Bemben MG (1998) Age-related alterations in muscular endurance. Sports Med 25:259-269. doi:10.2165/00007256-19982504000004

Bemben MG, Massey BH, Bemben DA, Misner JE, Boileau RA (1996) Isometric intermittent endurance of four muscle groups in men aged 20-74 years. Med Sci Sports Exerc 28:145-154. doi:10.1097/00005768-199601000-00026

Biering-Sørensen F (1984) Physical measurements as risk indicators for low-back trouble over a one-year period. Spine 9:106-119. doi:10.1097/00007632-198403000-00002

Borg G (1990) Psychophysical scaling with applications in physical work and the perception of exertion. Scand J Work Environ Health 16(Suppl 1):55-58

Brach JS, Simonsick EM, Kritchevsky S, Yaffe K, Newman AB (2004) The association between physical function and lifestyle activity and exercise in the health, aging and body composition study. J Am Geriatr Soc 52:502-509. doi:10.1111/j.1532-5415.2004. 52154.x

Cohen J (2003) Applied multiple regression: correlation analysis for the behavioral sciences, 3rd edn. Erlbaum, London

De Zwart BC, Frings-Dresen MH, Van Dijk FJ (1995) Physical workload and the aging worker: a review of the literature. Int Arch Occup Environ Health 68:1-12. doi:10.1007/BF01831627

De Zwart BC, Broersen JP, Frings-Dresen MH, Van Dijk FJ (1997) Musculoskeletal complaints in The Netherlands in relation to age, gender and physically demanding work. Int Arch Occup Environ Health 70:352-360

Era P, Schroll M, Hagerup L, Schultz-Larsen JK (2001) Changes in bicycle ergometer test performance and survival in men and women from 50 to 60 and from 70 to 80 years of age: two longitudinal studies in the Glostrup (Denmark) population. Gerontology 47:136-144. doi:10.1159/000052787

Hamberg-van Reenen HH, Ariëns GAM, Blatter BM, Twisk JW, Van Mechelen W, Bongers PM (2006) Physical capacity in relation to low back, neck, or shoulder pain in a working population. Occup Environ Med 63:371-377. doi:10.1136/oem.2006.026914

Hunter SK, Critchlow A, Enoka RM (2005) Muscle endurance is greater for old men compared with strength-matched young men. J Appl Physiol 99:890-897. doi:10.1152/japplphysiol.00243.2005

Ilmarinen JE (2001) Aging workers. Occup Environ Med 58:546-552. doi:10.1136/oem.58.8.546

Izquierdo M, Hakkinen K, Anton A, Garrues M, Ibanez J, Ruesta M, Gorostiaga EM (2001) Maximal strength and power, endurance performance, and serum hormones in middle-aged and elderly men. Med Sci Sports Exerc 33:1577-1587. doi:10.1097/0000 5768-200109000-00022

Lusa S, Louhevaara V, Kinnunen K (1994) Are the job demands on physical work capacity equal for young and aging firefighters? J Occup Med 36:70-74

Macaluso A, De Vito G (2004) Muscle strength, power and adaptations to resistance training in older people. Eur J Appl Physiol 91:450472. doi:10.1007/s00421-003-0991-3

Rantanen T, Sipila S, Suominen H (1993) Muscle strength and history of heavy manual work among elderly trained women and randomly chosen sample population. Eur J Appl Physiol Occup Physiol 66:514-517. doi:10.1007/BF00634301

Savinainen M, Nygard CH, Ilmarinen J (2004a) A 16-year follow-up study of physical capacity in relation to perceived workload among ageing employees. Ergonomics 47:1087-1102. doi:10. 1080/00140130410001686357

Savinainen M, Nygard CH, Korhonen O, Ilmarinen J (2004b) Changes in physical capacity among middle-aged municipal employees over 16 years. Exp Aging Res 30:1-22. doi:10.1080/036107 3049025746

Seitsamo J, Klockars M (1997) Aging and changes in health. Scand J Work Environ Health 23(Suppl 1):27-35

Sluiter JK (2006) High-demand jobs: age-related diversity in work ability? Appl Ergon 37:429-440. doi:10.1016/j.apergo.2006. 04.007

Statistics Netherlands http://statline.cbs.nl. Cited 21 Dec 2006

Twisk J (2003) Applied longitudinal data analysis for epidemiology. A practical guide. University Press, Cambridge

Van der Grinten MP (1992) Development of a practical method for measuring body part discomfort. In: Kumar S (ed) Advances in industrial ergonomics and safety, 4th edn. Taylor \& Francis, London

World Health Organization (1993) Aging and working capacity. Report of a WHO study group. World Health Organ Tech Rep Ser 835:1-49 